\title{
Estratos de risco e qualidade do cuidado à pessoa idosa na Atenção Primária à Saúde*
}

\author{
Líliam Barbosa Silva1, 2 \\ (iD) https://orcid.org/0000-0002-5576-9744 \\ Patrícia Aparecida Barbosa Silva ${ }^{1}$ \\ (iD) https://orcid.org/0000-0001-7363-0773 \\ Joseph Fabiano Guimarães Santos ${ }^{3}$ \\ (D) https://orcid.org/0000-0002-2001-996X \\ Salete Maria de Fátima Silqueira ${ }^{1}$ \\ (iD) https://orcid.org/0000-0002-4248-7107 \\ Eline Lima Borges ${ }^{1}$ \\ (D) https://orcid.org/0000-0002-0623-5308 \\ Sônia Maria Soares ${ }^{1}$ \\ (iD) https://orcid.org/0000-0003-3161-717X
}

Objetivo: identificar padrões de associações entre o grau de conformidade às solicitações de exames laboratoriais por estratos de risco e os parâmetros de resultados da qualidade do cuidado na Atenção Primária à Saúde (APS). Método: estudo transversal envolvendo 108 idosos com hipertensão e/ou diabetes atendidos na APS. Utilizaram-se questionário semiestruturado e dados de prontuário eletrônico. Para avaliação da qualidade do cuidado, utilizou-se o questionário Patient Assessment of Chronic Illness Care (PACIC). Realizaram-se análise descritiva, análise de correspondência múltipla e agrupamento k-means. Resultados: observou-se baixa conformidade da prática assistencial, destacando-se como pior parâmetro a avaliação do pé diabético (2,2\%). Foram identificados três clusters, sendo que o cluster 1 obteve maior número de indivíduos $(37,0 \%)$, com melhores indicadores da qualidade do cuidado, evidenciado pelo cumprimento dos exames laboratoriais acima de $50 \%(75,0 \%)$, escore PACIC elevado $(47,2 \%)$, controle dos níveis pressóricos $(70,0 \%)$ e metabólico $(95,0 \%)$, e satisfação com a saúde $(92,5 \%)$ e com o acesso à saúde $(90,0 \%)$. Em oposição, o cluster 3 $(29,6 \%)$ foi constituído por indivíduos com piores indicadores de resultado do cuidado. Conclusão: constataram-se baixa conformidade da prática assistencial e assimetrias entre oferta de ações em saúde e necessidades dos usuários, indicando falhas no processo de cuidado na APS.

Descritores: Registros Eletrônicos de Saúde; Qualidade da Assistência à Saúde; Saúde do Idoso; Atenção Primária à Saúde; Doença Crônica; Estudos Transversais.

\section{Como citar este artigo}

Silva LB, Silva PAB, Santos JFG, Silqueira SMF, Borges EL, Soares SM. Risk strata and quality of care for the elderly in Primary Health Care. Rev. Latino-Am. Enfermagem. 2019;27:e3166. [Access Available in: DOI: http://dx.doi.org/10.1590/1518-8345.2968.3166.

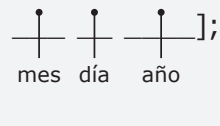




\section{Introdução}

O diabetes mellitus (DM) e a hipertensão arterial (HA) se configuram atualmente entre as condições crônicas mais prevalentes. Em conjunto, são consideradas como principais causas primárias de doenças renocardiovasculares no cenário mundial(1) e responsáveis por 13,0 milhões de mortes no mundo, em 2015, das quais 7,8 milhões ocasionadas pelas complicações dos níveis pressóricos elevados e 5,2 milhões atribuíveis ao descontrole metabólico(2).

Esse cenário é alarmante e sugere a necessidade de investir em melhorias na qualidade do manejo dessas morbidades, principalmente na Atenção Primária à Saúde (APS), por ser o nível de atenção responsável pela deteç̧ão precoce das condições crônicas e acompanhamento daqueles já diagnosticados, evitando suas complicações e a necessidade de internação.

Particularmente, no Brasil, foi adotado pelo Ministério da Saúde o Plano de Ações Estratégicas para o Enfrentamento das Doenças Crônicas Não Transmissíveis (DCNT) no Brasil 2011-2022(3) como norteador das ações direcionadas ao cuidado à pessoa que vivencia uma condição crônica de saúde. A porta de entrada ao sistema de saúde público brasileiro (Sistema Único de Saúde - SUS) consiste na Estratégia Saúde da Família que tem como foco o cuidado de saúde primário, centrado na família e na comunidade ${ }^{(4-7)}$. Sua principal função é prover cuidado centrado na pessoa, com prioridade em ações de promoção da saúde, prevenção de doenças, vigilância em saúde, assistência e acompanhamento da população adscrita, na tentativa de impactar os resultados da qualidade do cuidado provido aos usuários com condições crônicas na APS(3).

Algumas das estratégias recomendadas nesse intuito diz respeito à implementação de práticas clínicas baseadas em evidências, assim como o monitoramento dessas intervenções. Contudo, apesar de haver elevado grau de concordância sobre as melhores práticas de prevenção, diagnóstico e tratamento do DM e da HA, descritas em diversas diretrizes clínicas e protocolos, o seu uso ainda é incipiente, sobretudo quando se refere à implementação de ações segundo estratificação de risco da população abordada, tornando-se barreira para alcançar melhores resultados assistenciais com base no conhecimento científico vigente (8-11). $^{-}$

Ilustrando essa situação, uma pesquisa brasileira sobre a qualidade do cuidado à pessoa com DM em uma unidade básica de saúde do município de Ribeirão Preto, São Paulo, com base em 138 prontuários, revelou que as ações implementadas na assistência se encontram fragmentadas: não foi encontrado nenhum registro de índice de massa corporal; a avaliação dos pés e oftalmológica foi registrada em somente 15,2\% e 4,3\% dos prontuários, respectivamente; e entre os resultados de exames laboratoriais, apenas $1,4 \%$ tinha registrado valores de microalbuminúria(12).

Esses resultados provavelmente seriam mais alarmantes, caso fosse considerada a oferta dos serviços segundo as necessidades das pessoas abordadas. Esta era uma preocupação já manifestada pelo estudioso do assunto(13) que alertava para a possibilidade de que os usuários com maior risco clínico poderiam não estar sendo priorizados na oferta de ações às suas necessidades, visto que os sistemas de saúde ainda elegem o planejamento da oferta em detrimento das necessidades que é diferenciada segundo estratificação de seus riscos. Tal aspecto merece atenção, visto que, como consequência, pode-se empreender esforços e recursos em intervenções desnecessárias, equivocadas e ineficientes, fato este que merece ser melhor explorado.

Mediante o exposto, a hipótese da pesquisa é que existem assimetrias entre oferta de ações em saúde pelas equipes de Saúde da Família e necessidades dos idosos com DM e/ou HA, apresentando quadro menos favorável para o segmento com piores resultados do cuidado.

Ressalta-se que, até o momento, não se conhece trabalhos prévios que tenham investigado a associação entre os resultados da qualidade do cuidado e a conformidade dos provedores aos protocolos de cuidado a idosos com DM e/ou HA, considerando a solicitação de exames laboratoriais por estratificação de risco dos indivíduos.

Logo, o objetivo deste estudo foi identificar padrões de associações entre o grau de conformidade às solicitações de exames laboratoriais por estratos de risco cardiovascular e controle metabólico, bem como os parâmetros de resultados da qualidade do cuidado prestado ao idoso com condições crônicas na APS.

\section{Método}

Esta pesquisa de delineamento transversal faz parte da segunda etapa do estudo "Envelhecimento e Doença Renal" (en-DoRen), de base populacional em âmbito regional, realizado no período de agosto de 2014 a janeiro de 2017, em um dos nove distritos sanitários de Belo Horizonte, Minas Gerais, Brasil(14).

A amostra da linha de base do estudo en-DoRen é constituída por 300 idosos aleatorizados. Nessa análise, consideraram-se os dados dos participantes que atenderam aos seguintes critérios de inclusão: ter idade $\geq 60$ anos; autorrelatar DM e/ou HA ou ter diagnóstico confirmado em prontuário eletrônico; ser acompanhado por pelo menos um ano pela equipe de Saúde da Família do referido distrito; ter frequentado 
algum tipo de serviço de saúde da unidade básica de saúde (UBS) nos últimos três anos; ter sido localizado o prontuário eletrônico. Foram excluídos da análise os idosos com incapacidade cognitiva grave (Mini-Exame do Estado Mental - MEEM $\leq$ 9).

O tamanho amostral foi baseado em critério conservador, adotando prevalência de $50 \%$ dos diferentes desfechos (parâmetros assistenciais por estrato de risco cardiovascular e controle metabólico), o qual delimita a máxima variabilidade do tamanho amostral. Utilizando o método de cálculo proposto por Lwanga e Lemeshow ${ }^{(15)}$ e considerando precisão de estimativa absoluta de $10 \%$, com nível de significância de $5 \%$, foi encontrado tamanho amostral de 96 indivíduos. Adotando 10\% para possíveis perdas, o número total foi de 106 pessoas.

$\mathrm{Na}$ linha de base do estudo en-DoRen, identificaramse 143 usuários da APS, dos quais 118 idosos possuíam diagnóstico de DM e/ou HA. Destes, um total de 10 participantes foram excluídos da análise devido a não localização do prontuário eletrônico $(n=1)$ e tempo superior a três anos da última visita à UBS $(n=9)$. Após o cumprimento dos critérios de inclusão e exclusão, a amostra foi delimitada em 108 idosos.

A coleta de dados envolveu inquérito domiciliar, conduzido por duas das autoras e seis bolsistas de iniciação científica, previamente treinadas. Utilizouse questionário semiestruturado e pré-testado, contendo dados sociodemográficos (sexo, idade, escolaridade, renda), comportamental (tabagismo), clínicos (morbidades autorreferidas, MEEM), níveis de satisfação (satisfação com a saúde e satisfação com o acesso aos serviços de saúde) e qualidade do cuidado na APS (escores Patient Assessment of Chronic Illness Care - PACIC), em sua versão traduzida e adaptada para a língua portuguesa(16). Dados antropométricos foram obtidos no domicílio e, posteriormente, calculado o índice de massa corporal. Também foram aferidos níveis pressóricos e coletado material biológico para dosagem do perfil glicêmico, lipídico e parâmetros da função renal. Maiores detalhes se encontram descritos em publicação prévia(14).

Após inquérito domiciliar, foram coletados, em janeiro de 2017, dados secundários de prontuários eletrônicos. Em um impresso padrão foram registradas informações referentes às morbidades; parâmetros assistenciais, incluindo número de consultas médica e de enfermagem e exames (eletrocardiograma, fundoscopia, radiografia de tórax e avaliação do pé diabético); data de solicitação dos exames laboratoriais de interesse, bem como aqueles exames solicitados, mas que por algum motivo não foram realizados.

A delimitação do período para análise das informações dos prontuários considerou a periodicidade recomendada pelo protocolo de DM e HA estabelecida pela Secretaria de Estado de Saúde de Minas Gerais( ${ }^{(17)}$.

Os procedimentos de interesse exames laboratoriais foram considerados realizados se na aba "Procedimento" do prontuário eletrônico estivesse registrado o exame dentro do intervalo de tempo preconizado, considerando a estratificação de risco do participante.

A classificação de risco cardiovascular global foi categorizada em baixo, moderado e alto risco, conforme protocolo da Secretaria Municipal de Saúde de Belo Horizonte, Minas Gerais ${ }^{(18)}$, pela sua simplicidade e facilidade de adequação aos recursos do município.

Particularmente, o grau de conformidade às solicitações de exames laboratoriais foi calculado dividindo a soma dos exames laboratoriais em conformidade com o protocolo supracitado(17) pelo número total de exames recomendados e expressando-o como uma porcentagem. Para as pessoas com $\mathrm{HA}$, o número total de exames recomendados correspondeu a nove e para pessoas com DM, dez. Posteriormente, foram categorizados em: 0\% (E1) - (nenhum exame cumprido); 1-50\% (E2) (1 a 4 exames cumpridos para HA e 1 a 5 exames cumpridos para DM); $51-100 \%$ (E3) (5 a 9 exames cumpridos para HA e 6 a 10 exames cumpridos para DM).

A qualidade do cuidado foi amparada conceitualmente em duas dimensões donabedianas ${ }^{(19)}$ : processo - consistiu no registro dos parâmetros assistenciais pelo médico e pelo enfermeiro; no grau de conformidade às solicitações de exames laboratoriais segundo estrato de risco; e no número de exames laboratoriais solicitados e não realizados; e resultado - envolveu indicadores clínicos (controle dos níveis pressórico e metabólico); percepção do usuário sobre a qualidade do cuidado recebido na APS (escore PACIC); grau de satisfação com a saúde e com o acesso aos serviços de saúde; e meta terapêutica alcançada (resultados de exames laboratoriais coletados no domicílio).

Os parâmetros bioquímicos foram classificados dentro da faixa de normalidade e considerados para análise da meta terapêutica alcançada: creatinina sérica ( $<1,3 \mathrm{mg} / \mathrm{dL}$ em homens e $<1,2 \mathrm{mg} / \mathrm{dL}$ em mulheres), microalbuminúria (razão albumina/creatinina ACR $<30 \mathrm{mg} / \mathrm{g}$ ), glicemia sérica em jejum (< 100 $\mathrm{mg} / \mathrm{dL}$ ), colesterol total ( $<200 \mathrm{mg} / \mathrm{dL}$ ), high density lipoprotein cholesterol (HDL-c $\geq 40 \mathrm{mg} / \mathrm{dL}$ em homens e $\geq 50 \mathrm{mg} / \mathrm{dL}$ em mulheres), low density lipoprotein cholesterol (LDL-c $<160 \mathrm{mg} / \mathrm{dL}$ ), triglicérides ( $<150 \mathrm{mg} / \mathrm{dL})$, potássio $(\leq 5,1 \mathrm{mEq} / \mathrm{L})$, hematócrito (40-50\% em homens e $36-46 \%$ em mulheres), urina rotina (ausência de elementos anormais e infecção urinária) e hemoglobina glicada (HbA1c < 7\% em idosos com DM e <6,5\% em idosos com HA). 
Pressão arterial controlada (PA-c) foi definida como níveis pressóricos $<140 / 90 \mathrm{mmHg}$ em idosos com HA e $<130 / 80 \mathrm{mmHg}$ em idosos com DM; para pressão arterial elevada (PA-e), consideraram-se valores maiores ou iguais aos supracitados. Já controle metabólico controlado $(\mathrm{CM}-\mathrm{c})$ foi considerado $\mathrm{HbA} 1 \mathrm{c}<7 \%$ em idosos diabéticos e $<6,5 \%$ em idosos hipertensos, enquanto idosos com valores fora dos valores de referência supracitados foram considerados como controle metabólico alterado (CM-a).

O grau de satisfação com a saúde e com o acesso aos serviços de saúde foi mensurado pelas questões número dois e 24 do questionário World Health Organization Quality of Life-bref (WHOQOL-bref), versão traduzida e validada para o idioma português(20), respectivamente. Considerou-se satisfeito aqueles indivíduos que responderam estar "(muito) satisfeito" (satisfeito com a saúde [SS-s] e satisfeito com o acesso [SA-s]) e insatisfeito aqueles que se encontravam "(muito) insatisfeito" ou "razoável" (insatisfeito com a saúde [SS-i] e insatisfeito com o acesso [SA-i]).

Os escores PACIC foram categorizados de acordo com os percentis 25, 50 e 75, respectivamente: baixo ( $P-b \leq 1,30)$, médio $(P-m=1,31-2,22)$ e alto $(P-a \geq 2,23)$.

Inicialmente, os dados foram analisados por meio de técnicas descritivas expressas como proporções ou porcentagens para as variáveis categóricas e mediana (intervalo interquartílico - IQ) para as variáveis contínuas não paramétricas.

Para identificação de padrões de associação entre o grau de conformidade às solicitações de exames laboratoriais e os parâmetros de resultados da qualidade do cuidado na APS, utilizou-se a Análise de Correspondência Múltipla (ACM). Essa técnica de análise consiste na projeção gráfica das variáveis ativas que configura os perfis dos clusters e a variável suplementar que identifica a disposição dos clusters em um plano multidimensional (tipologia). Ao mesmo tempo em que se procuram os eixos estruturantes, é essencial a observação dos efeitos da interdependência entre as categorias das variáveis quando se cruzam os eixos. A partir da análise relacional, pode-se verificar, no mesmo espaço, padrões de associações, traduzido pela definição de diversos núcleos de homogeneidade. A interpretação gráfica dos pontos possibilita dizer que a proximidade entre os pontos reflete similaridade ou associação, enquanto o distanciamento dos pontos é considerado não similar(21).

A execução da ACM se baseou na estrutura de matriz de presenças-ausências dos dados, em que se dispõem os $n$ indivíduos (linhas da matriz), caracterizados segundo $m$ atributos, ou seja, as variáveis de interesse (coluna da matriz). Cada eixo do gráfico explica uma porcentagem da variabilidade total dos dados (inércia) ${ }^{(21)}$. Para o processo interpretativo da ACM, seguiram-se os passos descritos por Carvalho(21).

O número de dimensões retidas foi determinado pela magnitude dos autovalores e da inércia, retendo as dimensões com valores mais elevados, ou seja, o ponto a partir do qual a variância deixa de apresentar descidas acentuadas, que, no estudo, corresponderam às duas primeiras dimensões. A seleção das variáveis para cada dimensão elegida foi orientada pelo valor da medida de discriminação igual ou superior ao valor da inércia. O critério de seleção das categorias das variáveis para cada eixo estruturante se baseou nos valores das quantificações (coordenadas e contribuições). Como o somatório das contribuições para cada dimensão é 1 , tomou-se como valor de referência a contribuição média, no caso 0,0714 ( $1 / 14$ categorias). A confiabilidade interna das dimensões retidas foi calculada pelo coeficiente alfa de Cronbach.

Em adição, análise de clusters pelo método de agrupamento não hierárquico ( $K$-means Cluster) foi requerida a fim de delimitar agrupamentos de indivíduos com características semelhantes. Tal técnica utilizou as coordenadas dos escores (OBject SCOres OBSCO) determinados pela ACM nas dimensões mais representativas, identificando clusters mutuamente excludentes por meio do cálculo da distância euclidiana quadrática (coeficiente de semelhança) das categoriasponto. A validação do número de clusters obtido foi comprovada pela análise de clusters hierárquica por meio de dois critérios de agrupamento distintos (método de Ward e critério do vizinho mais próximo). A representação gráfica dos coeficientes de fusão, de ambos os métodos, indicou queda acentuada das distâncias entre os coeficientes até o terceiro cluster, sendo considerado como ponto de corte ótimo(21).

Os parâmetros assistenciais cumpridos (consultas médica e de enfermagem; avaliação do pé diabético; exames eletrocardiograma, fundoscopia e radiografia de tórax) foram excluídos da ACM devido à provável subnotificação dos registros nos prontuários, o que poderia comprometer os resultados. A variável risco cardiovascular também não entrou na ACM, considerando existência de multicolinearidade entre as variáveis que compõem a estratificação de risco cardiovascular (controle dos níveis pressórico e metabólico). A retirada dessa variável no modelo da ACM resultou no aumento dos valores da inércia e das quantificações das duas dimensões elegidas, obtendo-se um padrão de relação entre as demais variáveis mais consistente. Para execução das análises, utilizou-se o programa Statistical Package for the Social Sciences (SPSS, versão 23.0, Chicago, IL, USA). 
O projeto foi aprovado pelos Comitês de Ética em Pesquisa das instituições envolvidas (Parecer n 1.238 .099 e Parecer no 1.351.378), atendendo aos trâmites legais. Obteve-se o Termo de Consentimento Livre e Esclarecido de cada participante e foram garantidos o sigilo dos dados e o anonimato.

\section{Resultados}

A amostra do estudo foi constituída por 108 idosos, com idade mediana de 71,5 anos (IQ 66,0-81,0 anos), predomínio do sexo feminino ( $\mathrm{n}=75 ; 69,4 \%)$, com até quatro anos de estudo $(n=62 ; 57,4 \%)$, e $31,5 \%(n=34)$ contavam com apenas um salário mínimo ou menos para provimento do domicílio. Mais da metade dos participantes ( $n=58 ; 53,7 \%$ ) referiu ter cinco ou mais morbidades, sendo as mais prevalentes dislipidemia ( $n=94 ; 87,0 \%$ ) e osteoartrose ( $n=47 ; 43,5 \%)$. Todos os participantes tinham HA $(n=108)$ e $42,6 \%(n=46)$ possuíam DM associada (dados não mostrados).

O maior percentual obtido em relação ao cumprimento das recomendações estabelecidas no protocolo referente aos parâmetros assistenciais foi o item consulta médica, com percentual superior a $50 \%$ ( $n=56$ ). Todos os outros itens tiveram porcentagens inferiores a $30 \%$, sendo o pior parâmetro o item pé diabético ( $n=1 ; 2,2 \%$ ). Risco cardiovascular elevado esteve presente em mais de $3 / 4$ da amostra ( $n=84$ ). Níveis pressórico e metabólico se encontravam controlados em 56,5\% ( $n=61)$ e 76,9\% ( $n=83)$ dos casos, respectivamente. Observou-se também que mais da metade dos participantes encontravase (muito) satisfeita com a saúde ( $n=62 ; 59,0 \%$ ) e com o acesso aos serviços de saúde ( $n=65 ; 61,9 \%$ ), entretanto pior percepção da qualidade do cuidado (Tabela 1), com escore PACIC mediano de 1,55 (IQ 1,30-2,23) (dado não mostrado).

Tabela 1 - Distribuição dos parâmetros assistenciais e dos resultados do cuidado à pessoa idosa com hipertensão arterial e/ou diabetes mellitus acompanhada na Atenção Primária à Saúde. Belo Horizonte, MG, Brasil, 2014-2017

\begin{tabular}{llc}
\hline Variáveis & $\mathbf{n}$ & $\%$ \\
\hline Parâmetros assistenciais cumpridos & 56 & 51,9 \\
Consulta médica & 27 & 25,0 \\
Consulta de enfermagem & 31 & 28,7 \\
Exame eletrocardiograma & 10 & 9,3 \\
Exame fundoscopia & 10 & 9,3 \\
Exame radiografia de tórax & 1 & 2,2 \\
Avaliação pé diabético* & & \\
Risco cardiovascular & 14 & 13,0 \\
Baixo & 10 & 9,2 \\
Médio & 84 & 77,8 \\
Alto & $($ continua...)
\end{tabular}

Tabela 1 - continuação

\begin{tabular}{|c|c|c|}
\hline Variáveis & $\mathrm{n}$ & $\%$ \\
\hline \multicolumn{3}{|l|}{ 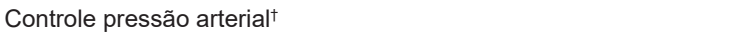 } \\
\hline Controlada & 61 & 56,5 \\
\hline Não controlada & 47 & 43,5 \\
\hline \multicolumn{3}{|l|}{ Controle metabólico ${ }^{\ddagger}$} \\
\hline Controlado & 83 & 76,9 \\
\hline Não controlado & 25 & 23,1 \\
\hline \multicolumn{3}{|l|}{ Satisfação com a saúde§ } \\
\hline Muito satisfeito / satisfeito & 62 & 59,0 \\
\hline Nem satisfeito nem insatisfeito & 28 & 26,7 \\
\hline Muito insatisfeito / insatisfeito & 15 & 14,3 \\
\hline \multicolumn{3}{|c|}{ Satisfação com o acesso aos serviços de saúde§ } \\
\hline Muito satisfeito / satisfeito & 65 & 61,9 \\
\hline Nem satisfeito nem insatisfeito & 17 & 16,2 \\
\hline Muito insatisfeito / insatisfeito & 23 & 21,9 \\
\hline \multicolumn{3}{|l|}{$\%$ exames cumpridos } \\
\hline $0 \%$ & 35 & 32,4 \\
\hline $1-50 \%$ & 24 & 22,2 \\
\hline $51-100 \%$ & 49 & 45,4 \\
\hline \multicolumn{3}{|l|}{ Escores PACICliा } \\
\hline Baixo $(\leq 1,30)$ & 29 & 28,7 \\
\hline Médio $(1,31-2,22)$ & 47 & 46,5 \\
\hline Alto $(\geq 2,23)$ & 25 & 24,8 \\
\hline
\end{tabular}

$*_{n}=46$ (somente pessoas com diabetes mellitus); ${ }^{+}$Pressão arterial controlada $=<140 / 90 \mathrm{mmHg}$ (idosos com hipertensão arterial) e $<130 / 80$ $\mathrm{mmHg}$ (idosos com diabetes mellitus); ${ }^{\ddagger}$ Controle metabólico controlado $=$ hemoglobina glicada $<7 \%$ (idosos com diabetes mellitus) e $<6,5 \%$

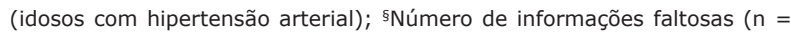
3); "Número de informações faltosas ( $n=7)$; "PACIC - Patient Assessment of Chronic Illness Care

Analisando o cumprimento das ações previstas no protocolo referente aos exames laboratoriais, foi identificado que 12 indivíduos $(11,1 \%)$ estavam em acordo com todas as recomendações (dado não mostrado), ao passo que $35(32,4 \%)$ não cumpriram nenhum dos requisitos (Tabela 1). Ainda, os exames creatinina sérica, urina rotina, potássio e microalbuminúria foram os analitos com menores percentuais de conformidade em ambos os casos (HA e DM) (abaixo de 50\%), e HbA1c, glicemia sérica em jejum e glicemia pós-prandial para as pessoas com DM com percentuais nulo a 10,9\%. Entre os exames solicitados e não realizados pelos participantes, destacaram-se microalbuminúria para pessoas com HA e glicemia pósprandial para aqueles com DM, apresentando percentuais de $38,2 \%(n=41)$ e $71,4 \%(n=33)$, respectivamente. Ainda, o pior indicador com meta terapêutica alcançada abaixo de $50 \%$ em ambos os casos foi evidenciado para o analito colesterol frações, ao passo que creatinina sérica e potássio apresentaram melhores metas terapêuticas alcançadas (Figura 1). 
(a) Pessoas idosas com hipertensão $(n=108)$

\begin{tabular}{rr|c|c|} 
Potássio & 28,0 & 39,8 & 98,0 \\
Creatinina & 23,9 & 49,1 & 90,7 \\
albuminúria & 38,2 & 18,5 & 89,7 \\
\hline Hematócrito & 28,0 & 52,8 & 81,3 \\
\hline Urina rotina & 29,1 & 45,4 & 75,9 \\
lesterol total & 27,0 & 52,8 & 71,7 \\
\hline Triglicérides & 27,9 & 53,7 & 65,1 \\
\hline icemia jejum & 25,8 & 57,4 & 51,9 \\
\hline terol frações & 27,0 & 52,8 & 45,7
\end{tabular}

(b) Pessoas idosas com diabetes ( $n=46)$

\begin{tabular}{ll|l|l} 
Creatinina & 25,0 & 50,0 & 97,8
\end{tabular}

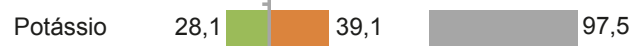

\begin{tabular}{ll|l|l|l} 
Microalbuminúria $\quad 27,8$ & 26,1 & 82,2
\end{tabular}

\begin{tabular}{ll|l|l|l|} 
Colesterol total & 25,0 & 60,9 & 68,9
\end{tabular}

\begin{tabular}{ll|l|l|} 
Urina rotina & 25,7 & 47,8 & 60,9
\end{tabular}

\begin{tabular}{ll|l|l|} 
Hemoglobina glicada $\quad 26,3$ & 6,5 & 52,2
\end{tabular}

\begin{tabular}{lr|r|r|} 
Triglicérides & 26,5 & 63,0 & 46,7
\end{tabular}

\begin{tabular}{ll|l|l|l|l|} 
Colesterol frações & 25,0 & 60,9 & 31,8
\end{tabular}

\begin{tabular}{c|c|c|c|} 
Glicemia jejum & 24,4 & 10,9 & 26,1 \\
Glicemia pós-prandial 71,4 & 0,0 &
\end{tabular}

$\begin{array}{lll}\text { Exame não } & \begin{array}{l}\text { Exame } \\ \text { cumprido }\end{array} \\ \text { realizado } & \text { alcançada }\end{array}$

Figura 1 - Distribuição de exames laboratoriais em conformidade com os parâmetros recomendados pelo protocolo de hipertensão arterial (a) e diabetes mellitus (b), exames não realizados e meta terapêutica alcançada. Belo Horizonte, MG, Brasil, 2014-2017
Em relação à $\mathrm{ACM}$, após avaliação gráfica da variância do número de dimensões máxima $\left(r_{\text {máx }}=11\right)$, decidiu-se reter as duas primeiras dimensões, as quais foram mais representativas em termos de inércia. A dimensão 1 (D1) explicou 19,94\% ([0,275/1,379]×100) e a dimensão 2 (D2), 17,26\% ([0,238/1,379]×100) da variância total. A confiabilidade interna da D1 e D2 foi 0,473 e 0,360, respectivamente. Os valores em negrito evidenciados na Tabela 2 correspondem às variáveis que melhor discriminaram em cada uma das duas dimensões em estudo. Todas registraram medidas de discriminação superiores à inércia da dimensão em referência.

A esse respeito, ressalta-se que as variáveis satisfação com a saúde e satisfação com o acesso à saúde, apesar de discriminarem menos que as demais variáveis em suas respectivas dimensões, optou-se por mantê-las na análise. A justificativa se respalda em três argumentos: primeiro, as medidas de discriminação e as quantificações (coordenadas e contribuições) de suas categorias não são valores praticamente nulos ou muito baixos em ambas as dimensões; segundo, a interpretação gráfica das medidas de discriminação (dados não mostrados) indica simultaneidade na composição das duas dimensões (situação de dupla pertença) por estarem localizadas próximas à diagonal e afastadas da origem; e terceira, uma interpretação mais qualitativa, em que a inclusão dessas variáveis contribuiu para a coerência temática na interpretação das dimensões.

Tabela 2 - Medidas de discriminação para as dimensões retidas e percentual de contribuição para cada variável em estudo. Belo Horizonte, MG, Brasil, 2014-2017

\begin{tabular}{|c|c|c|c|c|}
\hline \multirow[b]{2}{*}{ Variáveis } & \multicolumn{2}{|c|}{ Dimensão 1} & \multicolumn{2}{|c|}{ Dimensão 2} \\
\hline & $\begin{array}{c}\text { Medidas de } \\
\text { discriminação }\end{array}$ & $\begin{array}{c}\% \\
\text { contribuição }\end{array}$ & $\begin{array}{c}\text { Medidas de } \\
\text { discriminação }\end{array}$ & $\begin{array}{c}\% \\
\text { contribuição }\end{array}$ \\
\hline Controle metabólico* & 0,623 & 37,8 & 0,019 & 1,3 \\
\hline Controle da pressão arterial† & 0,429 & 26,0 & 0,129 & 9,0 \\
\hline Satisfação com o acesso à saúde & $\underline{0,222}$ & 13,5 & $\underline{0,115}$ & 8,0 \\
\hline Satisfação com a saúde & $\underline{0,187}$ & 11,3 & $\underline{0,210}$ & 14,7 \\
\hline PACIC $\ddagger$ total & 0,144 & 8,6 & 0,605 & 42,4 \\
\hline$\%$ exames cumpridos & 0,046 & 2,8 & 0,351 & 24,6 \\
\hline $\mathrm{N}^{\circ}$ de cluster§ & 0,711 & & 0,627 & \\
\hline Total ativo & 1,650 & & 1,428 & \\
\hline Inércia & 0,275 & & 0,238 & \\
\hline \% variância explicada & 19,94 & & 17,26 & \\
\hline Alpha Cronbach's & 0,473 & & 0,360 & \\
\hline
\end{tabular}

*Hemoglobina glicada controlada $=<7 \%$ (idosos com diabetes mellitus) e $<6,5 \%$ (idosos com hipertensão arterial); ${ }^{\dagger}$ Pressão arterial controlada $=<140 / 90$ $\mathrm{mmHg}$ (idosos com hipertensão arterial) e $<130 / 80 \mathrm{mmHg}$ (idosos com diabetes mellitus); ${ }^{\ddagger}$ PACIC = Patient Assessment of Chronic Illness Care; §Variável suplementar

Posteriormente, a análise das quantificações (coordenadas e contribuições) das categorias das variáveis selecionadas identificou que a D1 esteve associada com o controle satisfatório dos níveis pressóricos (PA-c) e metabólico (CM-c), e satisfação com sua saúde (SS-s) e com o acesso à saúde (SA-s) (D1 >0).
Em oposição, encontram-se as categorias controle insatisfatório dos níveis pressóricos (PA-e) e metabólico (CM-a), como também as categorias insatisfação com sua saúde (SS-i) e com o acesso à saúde (SA-i) $(D 1<0)$. Nesse sentido, a D1 separa os indivíduos quanto aos aspectos clínicos e níveis de satisfação. Em 
contrapartida, a D2 diferenciou os indivíduos com maior percentual de exames cumpridos (E3) e escores PACIC (P-a, P-m), assim como satisfação com a saúde (SS-s) e com o acesso à saúde (SA-s) (D2 > 0); opondo-se aos indivíduos com piores indicadores de resultado do cuidado (E1, E2, P-b, SS-i, SA-i) (D2 < 0) (Figura 2).

Em adição, a análise de agrupamento identificou a formação de três clusters de indivíduos com perfis distintos (Figura 2). O cluster 1 obteve maior número de indivíduos com 37,0\% $(\mathrm{n}=40)$ da amostra, seguido do cluster $2(\mathrm{n}=36 ; 33,3 \%)$ e cluster 3 ( $n=32 ; 29,6 \%)$. O primeiro cluster foi formado por indivíduos com melhores indicadores da qualidade do cuidado, evidenciado pelo cumprimento dos exames laboratoriais acima de 50\% (E3), escore PACIC elevado $(\mathrm{P}-\mathrm{a})$, controle dos níveis pressóricos (PA-c) e metabólico $(\mathrm{CM}-\mathrm{C})$, e satisfação com a saúde (SS-s) e com o acesso à saúde (SA-s). Em oposição, o cluster 3 foi constituído pela ausência completa no cumprimento das diretrizes (E1), escore mediano do PACIC (P-m), elevação dos níveis pressóricos (PA-e) e metabólico (CM-a), e insatisfação com a saúde (SS-i) e com o acesso à saúde (SA-i). O outro perfil evidenciado neste estudo (cluster 2) foi caracterizado por indivíduos com indicadores clínicos estáveis (PA-c e CM-c), entretanto com percentuais de cumprimento às diretrizes abaixo de 50\% (E1 e E2), baixo escore PACIC (P-b) e se encontram insatisfeitos com a saúde (SS-i) e com o acesso à saúde (SA-i) (Tabela 3 ).

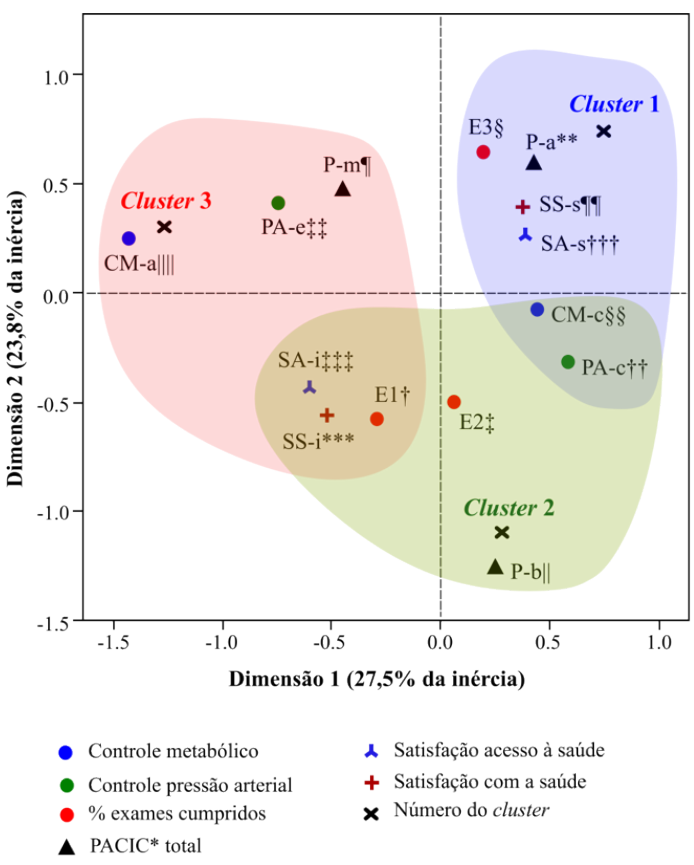

*PACIC $=$ Patient Assessment of Chronic Illness Care $;{ }^{+} \mathrm{E} 1$ = Exames cumpridos (0\%) $;{ }^{\ddagger} \mathrm{E} 2=$ Examescumpridos $(1-50 \%) ;{ }^{\mathrm{E}} \mathrm{E} 3=$ Exames cumpridos $(51-100 \%)$; "P-b = PACIC* baixo $(\leq 1,30) ;$ "P-m = PACIC* médio $(1,31-2,22)$; **P-a $=$ PACIC* alto $(\geq 2,23) ;{ }^{+} \mathrm{PA}-\mathrm{C}=$ Pressão arterial controlada ${ }^{\ddagger} \mathrm{PA}-\mathrm{e}=$ Pressão arterial elevada; ${ }^{\S \S} \mathrm{CM}-\mathrm{c}=$ Controle metabólico controlado; "IIICM-a = Controle metabólico alterado; "1"SS-s = Satisfeito com a saúde; ***SS-i = Insatisfeito com a saúde $;{ }^{++}$SA-s = Satisfeito com o acesso à saúde; ${ }^{\ddagger \neq}$ SA-i = Insatisfeito com o acesso à saúde

Figura 2 - Mapa de análise de correspondência múltipla segundo grau de conformidade às solicitações de exames laboratoriais e parâmetros de resultados da qualidade do cuidado à pessoa idosa com hipertensão arterial e/ou diabetes mellitus acompanhada na Atenção Primária à Saúde. Belo Horizonte, MG, Brasil, 2014-2017

Tabela 3 - Caracterização dos clusters segundo resultados do cuidado à pessoa idosa com hipertensão e/ou diabetes acompanhada na Atenção Primária à Saúde. Belo Horizonte, MG, Brasil, 2014-2017

\begin{tabular}{|c|c|c|c|c|c|c|c|}
\hline \multirow{3}{*}{ Variáveis } & & \multicolumn{6}{|c|}{ Tipologia } \\
\hline & & \multicolumn{2}{|c|}{ Cluster 1} & \multicolumn{2}{|c|}{ Cluster 2} & \multicolumn{2}{|c|}{ Cluster 3} \\
\hline & & $\mathbf{n}$ & $\%$ & $\mathbf{n}$ & $\%$ & $\mathbf{n}$ & $\%$ \\
\hline \multirow{4}{*}{$\%$ exames cumpridos } & $\mathrm{E} 1^{\dagger}(0 \%)$ & 7 & 17,5 & 15 & 41,7 & 13 & 40,6 \\
\hline & $E 2^{\ddagger}(1-50 \%)$ & 3 & 7,5 & 14 & 38,9 & 7 & 21,9 \\
\hline & E3§ $(51-100 \%)$ & 30 & 75,0 & 7 & 19,4 & 12 & 37,5 \\
\hline & Total & 40 & 100,0 & 36 & 100,0 & 32 & 100,0 \\
\hline \multirow{4}{*}{$\mathrm{PACIC}^{*}$ total } & $P-b^{\|}$ & 3 & 8,3 & 23 & 69,7 & 3 & 9,4 \\
\hline & P-m $\pi$ & 16 & 44,4 & 9 & 27,3 & 22 & 68,8 \\
\hline & $P-a^{* *}$ & 17 & 47,2 & 1 & 3,0 & 7 & 21,9 \\
\hline & Total & 36 & 100,0 & 33 & 100,0 & 32 & 100,0 \\
\hline \multirow{3}{*}{ Controle pressão arterial } & PA-c ${ }^{\dagger \dagger}$ & 28 & 70,0 & 30 & 83,3 & 3 & 9,4 \\
\hline & $P A-e^{\ddagger \ddagger}$ & 12 & 30,0 & 6 & 16,7 & 29 & 90,6 \\
\hline & Total & 40 & 100,0 & 36 & 100,0 & 32 & 100,0 \\
\hline \multirow{3}{*}{ Controle metabólico } & $\mathrm{CM}-\mathrm{C}^{\S \S}$ & 38 & 95,0 & 35 & 97,2 & 10 & 31,3 \\
\hline & CM-a & 2 & 5,0 & 1 & 2,8 & 22 & 68,8 \\
\hline & Total & 40 & 100,0 & 36 & 100,0 & 32 & 100,0 \\
\hline \multirow{3}{*}{ Satisfação com a saúde } & SS-STा & 37 & 92,5 & 14 & 41,2 & 11 & 35,5 \\
\hline & SS-i*** & 3 & 7,5 & 20 & 58,8 & 20 & 64,5 \\
\hline & Total & 40 & 100,0 & 34 & 100,0 & 31 & 100,0 \\
\hline \multirow{3}{*}{ Satisfação com o acesso à saúde } & SA-sttt & 36 & 90,0 & 15 & 44,1 & 14 & 45,2 \\
\hline & $S A-i \neq \nexists \neq$ & 4 & 10,0 & 19 & 55,9 & 17 & 54,8 \\
\hline & Total & 40 & 100,0 & 34 & 100,0 & 31 & 100,0 \\
\hline
\end{tabular}

${ }^{*} \mathrm{PACIC}=$ Patient Assessment of Chronic Illness Care. ${ }^{\dagger} \mathrm{E} 1$ = Exames cumpridos $(0 \%) ;{ }^{\dagger} \mathrm{E} 2$ = Exames cumpridos $(1-50 \%) ;{ }^{\mathrm{s}} \mathrm{E} 3=\mathrm{Exames}$ cumpridos $(51-100 \%) ; \| \mathrm{P}-\mathrm{b}=$ PACIC* baixo $(\leq 1,30) ;$ "P-m = PACIC* médio $(1,31-2,22)$; **P-a = PACIC* alto $(\geq 2,23)$; ${ }^{+\dagger}$ PA-c = Pressão arterial controlada;

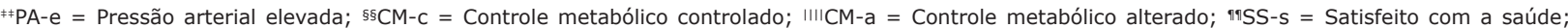

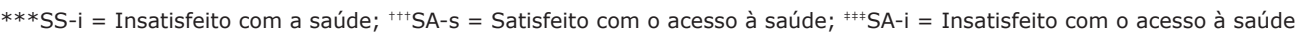




\section{Discussão}

Este estudo, envolvendo idosos com DM e/ou HA atendidos na APS, mostrou baixa conformidade da prática assistencial segundo estratificação de risco cardiovascular e controle metabólico, repercutindo na qualidade do cuidado alcançada. Ainda, foram identificados três clusters de indivíduos com perfis distintos, sendo o primeiro e o terceiro cluster formados por idosos que concentraram os melhores e os piores indicadores da qualidade do cuidado, respectivamente.

Mesmo com métodos distintos, que limitam a comparação entre os dados obtidos, outras pesquisas nacionais e internacionais também enfatizaram a incipiência da utilização do protocolo clínico de DM e HA recomendado para o aperfeiçoamento da assistência por médicos e enfermeiros atuantes na APS(10-11,22-23).

Nesse sentido, observa-se que desafios ainda precisam ser superados para garantir a melhoria do cuidado às pessoas com condições crônicas de saúde. Particularmente, sobre o cumprimento da periodicidade dos exames laboratoriais, o presente estudo identificou percentuais muito aquém do recomendado pelo protocolo, com destaque para aqueles relacionados ao perfil glicêmico em idosos com DM. Tal achado vai de encontro a um dos objetivos específicos da Rede de Atenção à Saúde das Pessoas com Doenças Crônicas que compreende propiciar o acesso aos recursos diagnósticos e terapêuticos adequados em tempo oportuno, garantindo-se a integralidade do cuidado, conforme a necessidade de saúde do usuário(24).

Em geral, houve maior cumprimento às recomendações referentes à $H A$, se comparado ao DM cuja maior proporção foi encontrada para o analito glicemia em jejum (cinco vezes mais). Esse dado indica que a integralidade do cuidado talvez ainda não constitua componente de excelência no atendimento dos usuários nas UBS investigadas, podendo ser um dos motivos pelo baixo percentual de pessoas com controle metabólico adequado, conforme constatado no estudo. Inclusive, ressalta-se que a glicemia pós-prandial não foi cumprida em nenhum dos casos investigados, não sendo, portanto, um exame de rotina de avaliação do perfil glicêmico e acompanhamento da terapêutica medicamentosa prescrita.

Ressalta-se que parte desse não cumprimento pode ser atribuído, inclusive, ao próprio absenteísmo dos idosos aos exames laboratoriais que merece ser discutido. Tal situação não apenas contribui para o crescimento progressivo da demanda reprimida, reduzindo a possibilidade de acesso, como também possibilita que haja aumento dos custos assistenciais, visto que o adiamento ou não realização dos exames solicitados pode provocar agravo da condição do usuário(25).
$\mathrm{Na}$ literatura, existem experiências exitosas sobre o monitoramento da taxa de absenteísmo, como aquelas que utilizam sistema de informação para identificar detalhadamente o usuário e a organização dos atendimentos. Ainda incluem monitoramento das metas assistenciais individuais e taxa de utilização dos serviços. Contudo, para que tal estratégia possa converter em resultados práticos, é necessário que o sistema de informação promova a agregação dos dados clínicos dos usuários, de modo a auxiliar o processo de planejamento, o reconhecimento de grupos de riscos com necessidades especiais e a gestão do cuidado integral, incluindo a emissão de avisos e alertas para a manutenção de vínculo com o usuário(23).

A respeito da consulta de enfermagem, verificouse baixo registro sistemático acerca dessa prática assistencial na maioria dos prontuários, estando aquém daquele definido pelo protocolo (minimamente realizada semestralmente) ${ }^{(17)}$. Como agravante, foi identificado centralização dos registros sobre a queixaconduta durante o acolhimento, com consequente encaminhamento para avaliação médica, sendo, portanto, subtraídas as habilidades e as atribuições específicas do enfermeiro no cuidado ao idoso que vivencia uma condição crônica de saúde conforme já constatado em outra pesquisa(22).

Esse achado pode ser resultante de distintos fatores, desde o processo de formação acadêmica do enfermeiro, que prioriza em seus currículos a consulta de enfermagem a crianças e gestantes, até mesmo as condições de trabalho, os registros de enfermagem incompletos e a postura do próprio profissional conforme evidenciado em estudos brasileiros ${ }^{(26-27)}$. Tal realidade pode contribuir para que a categoria de enfermagem perca espaço de atuação já conquistado dentro das equipes de Saúde da Família, com consequente recuo da prática clínica desse profissional no cuidado ao idoso, que estará cada vez mais a cargo do médico, reforçando, assim, o modelo médico-centrado.

Em adição, os resultados também apontaram problemas concernentes à consulta médica, apesar de apresentar melhores resultados quando comparados à consulta de enfermagem. Do total dos prontuários investigados, 34,3\% não continham nenhum ou apenas um registro de consulta médica (dado não mostrado), sendo que o recomendado pelo protocolo(17) é de duas a três consultas anuais, a depender do risco cardiovascular em pessoas com HA ou do controle metabólico em pessoas com DM.

Diante da realidade apresentada, observa-se potencial insuficiência na oferta ou mau gerenciamento no agendamento dessas consultas, indo de encontro às proposições para implementação da Rede de Atenção 
à Saúde, que recomenda a aproximação da vigilância epidemiológica com o cenário de ocorrência das condições crônicas, com vistas a fortalecer a estratificação por riscos. Assim, busca-se garantir a gestão baseada nas necessidades de saúde da população(28), atendendo a um dos mais importantes princípios do SUS, quais seja, a equidade(13).

Nesse sentido, é provável que ocorram lacunas na organização do processo de trabalho das equipes de Saúde da Família, de modo a oferecer uma assistência equânime e integral, acolhendo o usuário e vinculando-o aos serviços ofertados segundo suas reais necessidades. Isso porque quando não se estratifica por riscos uma dada população pode ocorrer suboferta de cuidados necessários às pessoas com maiores riscos e/ou sobreoferta de cuidados desnecessários àqueles com menores riscos produzindo, por consequência, uma atenção inefetiva e ineficiente. Esse problema explica, em grande parte, as dificuldades de abrir agenda na Estratégia Saúde da Família para atenção à saúde às pessoas com condições crônicas com cuidados que não agregam valor às pessoas ${ }^{(13)}$.

Também foi evidenciado no presente estudo baixo percentual nas solicitações de exames de imagem, não podendo ser descartada a presença de possíveis falhas na comunicação entre os pontos de atenção na rede, resultando em provável subregistro desses procedimentos, os quais são realizados em outros níveis de atenção. Tal assertiva carece de evidências, mas caso seja comprovada, há necessidade de a gestão dar prioridade a essa temática de modo a garantir a contrarreferência na rede, considerada essencial para - exercício da coordenação da atenção pela APS. Para alguns autores ${ }^{(13,22,28)}$, é essa comunicação entre os diferentes pontos de atenção da rede que dará racionalidade ao atendimento, evitando que exames sejam realizados em duplicidade ou que sejam solicitados desnecessariamente, otimizando recursos com impacto na resolubilidade.

Por sua vez, a identificação dos três clusters no presente estudo destacou que, apesar de ser amplamente reconhecida a importância de se padronizar a conduta clínica e terapêutica para pessoas com HA e DM no intuito de alcançar melhores resultados clínicos e funcionais, nem sempre isso pode ser evidenciado. Uma possível explicação decorre da própria complexidade do cuidado à pessoa que vivencia uma condição crônica de saúde, pois requer manejo de forma pró-ativa, contínua e integrada pelo sistema de atenção à saúde, pelos profissionais e pelos usuários/família para seu controle efetivo, eficiente e com qualidade ${ }^{(13)}$. Portanto, o cumprimento das recomendações constatadas no protocolo por si só não garante necessariamente melhores resultados conforme foi observado nos distintos clusters identificados no estudo.

Particularmente, os integrantes dos clusters 2 e 3 apresentaram baixo cumprimento das recomendações do protocolo, entretanto o segundo cluster aglomerou idosos com parâmetros clínicos controlados, ao contrário do terceiro cluster. Isso sugere presença de outras variáveis que possam contribuir para esse achado não contemplado na presente análise, por exemplo, maior concentração de idosos com melhor autocuidado no cluster 2 do que no cluster 3. Tal assertiva carece de evidências e demanda maiores investigações.

Os dados apresentados nesta pesquisa reforçam a importância de sensibilizar e qualificar as equipes de Saúde da Família acerca da melhoria da implementação de diretrizes na prática clínica em prol do maior controle de idosos com DM e/ou HA visando promover alinhamento da atenção à saúde com as necessidades de saúde da população situada nos diferentes estratos de riscos. Para tanto, incentiva-se a criação de parcerias com instituições de ensino superior que poderão servir de apoio, explorando o recurso do Telessaúde da região, que atualmente é coordenada por uma grande instituição de ensino superior da capital, viabilizando iniciativa de apoio e educação permanente, conforme destacado nas diretrizes da Política Nacional de Educação Permamente em Saúde(24).

Além disso, importante destacar o papel das unidades de atenção ambulatorial especializada ( $A A E$ ) que integram a rede cuja atuação não se limita apenas ao seu papel assistencial, mas também participam de funções de supervisão e educação permanente dos profissionais da APS. Nessa perspectiva, é necessário identificar quais profissionais têm essas habilidades desenvolvidas e, uma vez identificados, propiciar meios necessários para organizar a agenda desses profissionais de modo a garantir tempo e espaço para essas atividades. Experiências pregressas mostram que os profissionais especializados são fundamentais na educação permanente dos profissionais generalistas(13).

Ademais, este estudo poderá subsidiar a atuação do enfermeiro, motivando-o a desempenhar papel de articulador do processo de trabalho na Estratégia Saúde da Família, ajudando a repensar a forma de cuidado ao idoso que vivencia uma condição crônica de saúde, incentivando a atuação de uma equipe multidisciplinar, verdadeiramente comprometida com uma prática equitativa, integral e resolutiva. Nesse contexto, é imperativo que não apenas a enfermagem, mas todos os profissionais cumpram suas atribuições e competências privativas de maneira plena, de tal modo a serem registradas em prontuários, valorizando as informações contidas nesses documentos, melhorando, assim, a qualidade da informação gerada. 
Por fim, este estudo reforça a necessidade de institucionalizar políticas de avaliação em saúde como processo de transformação de práticas na APS e fortalecer aquelas já existentes no Brasil, como a Portaria n. 483, de $1^{\circ}$ de abril de 2014(24), que ao redefinir a Rede de Atenção à Saúde das Pessoas com Doenças Crônicas no SUS e estabelecer diretrizes para a organização das suas linhas de cuidado recomenda o monitoramento e a avaliação da qualidade dos serviços por meio de indicadores de estrutura, processo e desempenho que investiguem a efetividade e a resolutividade da atenção.

A realização de auditorias clínicas periódicas, assim como o feedback com os profissionais de saúde envolvidos no cuidado a partir da divulgação de informes com os dados e indicadores monitorados, é uma estratégia que pode sistematizar de forma efetiva essas políticas ${ }^{(29)}$.

Os resultados do estudo devem ser interpretados com cautela em razão do seu delineamento transversal, que impossibilita estabelecer relação temporal e causal entre os parâmetros assistenciais e a qualidade do cuidado na APS. Outra limitação se refere aos dados secundários dos prontuários eletrônicos, dependente da qualidade dos registros dos profissionais de saúde (viés de informação) e o tipo de análise aplicada, sem nenhum controle do fator de confundimento. Contudo, essas limitações não inviabilizam os achados que podem auxiliar gestores e profissionais de saúde no fortalecimento de políticas voltadas à avaliação da qualidade do cuidado na APS.

\section{Conclusão}

O estudo mostrou baixa conformidade da prática assistencial, destacando-se a avaliação do pé diabético e a solicitação de exames especializados, indicando falhas no processo de cuidado na APS. Verificouse também que a técnica de formação de clusters mostrou-se interessante enquanto ferramenta de gestão clínica, possibilitando identificar grupos distintos dentro de um mesmo serviço de saúde, direcionando, consequentemente, intervenções específicas.

Ainda foi possível confirmar a hipótese da existência de assimetrias entre oferta de ações em saúde pelas equipes de Saúde da Família e necessidades apresentadas pelos idosos com DM e/ou HA, apresentando quadro menos favorável para o segmento com piores resultados do cuidado.

\section{Referências}

1. Hu JR, Coresh J. The public health dimension of chronic kidney disease: what we have learnt over the past decade. Nephrol Dial Transplant. 2017 Apr 1;32(Suppl 2):ii113-ii20. doi: 10.1093/ndt/gfw416.
2. Benjamin EJ, Virani SS, Callaway CW, Chamberlain AM, Chang AR, Cheng S, et al. Heart Disease and Stroke Statistics - 2018 Update. A report from the American Heart Association. Circulation. 2018 Jan; 137:e67-e492. doi: 10.1161/CIR.0000000000000558.

3. Malta DC, Morais Neto OL, Silva Junior JB. Presentation of the strategic action plan for coping with chronic diseases in Brazil from 2011 to 2022. Epidemiol Serv Saúde. 2011 Oct/Dec;20(4):425-38. doi:10.5123/ S1679-49742011000400002.

4. Andrade MV, Coelho AQ, Xavier M Neto, Carvalho LR, Atun R, Castro MC. Brazil's Family Health Strategy: factors associated with programme uptake and coverage expansion over 15 years (1998-2012). Health Policy Plan. 2018 Apr 1;33(3):368-80. doi: 10.1093/heapol/czx189.

5. Carvalho VCHS, Rossato SL, Fuchs FD, Harzheim E, Fuchs SC. Assessment of primary health care received by the elderly and health related quality of life: a crosssectional study. BMC Public Health. 2013 Jun 24;13:605. doi:10.1186/1471-2458-13-605.

6. Furlanetto DL, Pinho DL, Parreira CM. Reorientation of human resources for health: a great challenge for the Brazilian National Health System. Public Health. 2015 Sept;129(9):1166-71. doi: 10.1016/j.puhe.2015.07.003.

7. Paim J, Travassos C, Almeida C, Bahia L, Macinko J. The Brazilian health system: history, advances, and challenges. Lancet. 2011 May 21;377(9779):1778-97. doi: 10.1016/S0140-6736(11)60054-8.

8. Adedeji AR, Tumbo J, Govender I. Adherence of doctors to a clinical guideline for hypertension in Bojanala district, North-West Province, South Africa. Afr J Prim Health Care Fam Med. 2015 Apr 13;7(1):776. doi: 10.4102/phcfm.v7i1.776.

9. Frølich A. Identifying organisational principles and management practices important to the quality of health care services for chronic conditions. Dan Med J. [Internet]. 2012 Feb [cited Jan 8, 2018];59(2):B4387. Available from: https://www.ncbi.nlm.nih.gov/pubmed/22293057 10. Kapongo RY, Lulebo AM, Mafuta EM, Mutombo PB, Dimbelolo JCM, Bieleli IE. Assessment of health service delivery capacities, health providers' knowledge and practices related to type 2 diabetes care in Kinshasa primary healthcare network facilities, Democratic Republic of the Congo. BMC Health Serv Res. 2015 Jan 22;15:9. doi: 10.1186/s12913-015-0679-5.

11. Lulebo AM, Mapatano MA, Kayembe PK, Mafuta EM, Mutombo PB, Coppieters Y. Assessment of hypertension management in primary health care settings in Kinshasa, Democratic Republic of Congo. BMC Health Serv Res. 2015 Dec 24;15:573. doi:10.1186/s12913-015-1236-y. 12. Silva ASB, Santos MA, Teixeira CRS, Damasceno MMC, Camilo J, Zanetti ML. [Evaluating diabetes mellitus care in a brazilian basic health district]. Texto Contexto Enferm. 2011 Jul/Sept;20(3):512-8. http://dx.doi. org/10.1590/S0104-07072011000300012 Portuguese. 
13. Mendes EV. A construção social da Atenção Primária à Saúde. Brasília: Conselho Nacional de Secretários de Saúde; 2015. 193 p. [Internet]. [cited June 4, 2017]. Available from: http://www.saude.go.gov.br/ wp-content/uploads/2016/12/a-construcao-social-daatencao-primaria-a-saude.pdf. [Portuguese].

14. Silva LB, Soares SM, Silva PAB, Santos JFG, Miranda LCV, Santos RM. Assessment of the quality of primary care for the elderly according to the Chronic Care Model. Rev. Latino-Am. Enfermagem. 2018;26:e2987. doi: 10.1590/1518-8345.2331.2987.

15. Naing NN. Determination of sample size. Malays J Med Sci. [Internet]. 2003 Jul [cited Jun 25, 2018];10(2):84-6. Available from: https://www.ncbi. nlm.nih.gov/pmc/articles/PMC3561892/

16. Schwab GL, Moysés ST, Kusma SZ, Ignácio SA, Moysés SJ. Perception of innovations in Chronic Diseases/Conditions' care: an evaluative research in Curitiba. Saúde Debate. [Internet]. 2014 Oct [cited Dec 2, 2018];38(special):307-18. Available from: http:// www.scielo.br/pdf/sdeb/ v38nspe/0103-1104-sdeb-38- spe-0307.pdf

17. Secretaria de Estado de Saúde de Minas Gerais SES/MG. Linha-guia de hipertensão arterial sistêmica, diabetes mellitus e doença renal crônica. 3. ed. Belo Horizonte: SES/MG; 2013. 200 p. (Atenção à Saúde do Adulto). [Internet]. [cited Mar 24, 2017]. Available from: http://www.saude.mg.gov.br/images/documentos/ guia_de_hipertensao.pdf. [Portuguese].

18. Pereira AF, Gontijo CC, Augusto DK, Rodrigues EAS, Reis GA, Lara HA, et al. Protocolo de hipertensão arterial / risco cardiovascular. Belo Horizonte: Secretaria Municipal de Saúde de Belo Horizonte; 2011. 71 p. [Internet]. [cited Apr 25, 2017]. Available from: https:// prefeitura.pbh.gov.br/sites/default/files/estrutura-degoverno/saude/2018/documentos/ Diabetes/protocolo_ hipertensao.pdf. [Portuguese].

19. Donabedian A. The quality of medical care. Science. 1978 May 26;200(4344):856-64. doi: 10.1126/science.417400. 20. Fleck MPA, Louzada S, Xavier M, Chachamovich E, Vieira G, Santos L, et al. Application of the Portuguese version of the abbreviated instrument of quality life WHOQOL-bref. Rev Saúde Pública. 2000 Apr;34(2):178-83. [Portuguese]. doi: 10.1590/S003489102000000200012.

21. Carvalho H. Análise multivariada de dados qualitativos: utilização da análise de correspondências múltiplas com o SPSS. 2. ed. Sílabo: Portugal; 2017. [Portuguese].

22. Silva ASB, Santos MA, Teixeira CRS, Damasceno MMC, Camilo J, Zanetti ML. Evaluating diabetes mellitus care in a brazilian basic health district. Texto Contexto Enferm. 2011 Jul/Sept;20(3):512-8. [Portuguese]. doi: $10.1590 /$ S0104-07072011000300012.

Autor correspondente:

Líliam Barbosa Silva

E-mail: ligemeasbh@yahoo.com.br

(iD) https://orcid.org/0000-0002-5576-9744
23. Santos CM, Barbieri AR, Gonçalves CCM, Tsuha DH. Evaluation of the healthcare network for persons with arterial hypertension: study of a health district. Cad Saude Pública. 2017;33(5):e00052816. [Portuguese]. doi: 10.1590/0102-311X00052816.

24. Portaria no 483, de $1^{0}$ de abril de 2014 (BR). Redefine a Rede de Atenção à Saúde das Pessoas com Doenças Crônicas no âmbito do Sistema Único de Saúde (SUS) e estabelece diretrizes para organização das suas linhas de cuidado. Diário Oficial da República Federativa do Brasil, Brasília (DF), 2014 Abr 1;Seção 1:50-52. [Internet]. [Acesso $23 \mathrm{dez} 2018$ ]. Disponível em: https://www.jusbrasil.com.br/diarios/68448289/ dou-secao-1-02-04-2014-pg-50. [Portuguese].

25. Cavalcanti RP, Cavalcanti JCM, Serrano RMSM, Santana PR. Absenteeism of specialized consultations in the public health system: relationship between causes and the work process of family health teams, João Pessoa - PB, Brazil. Tempus Actas Saúde Coletiva. 2013 Sept;7(2):63-84. [Portuguese]. doi: 10.18569/tempus.v7i2.1344.

26. Dantas CN, Santos VEP, Tourinho FSV. Nursing consultation as a technology for care in light of the thoughts of Bacon and Galimberti. Texto Contexto Enferm. 2016 Mar 22;25(1):e2800014. doi: 10.1590/0104-0707201500002800014.

27. Silva KM, Santos SMA. The nurse appointment to the elderly on family health strategy: challenges and possibilities. CiencCuid Saude. 2014Jan/Mar;13(1):49-57. doi: 10.4025/cienccuidsaude.v13i1.20128.

28. Santos RSAF, Bezerra LCA, Carvalho EF, Fontbonne A, Cesse EÂP. Health Care Network to people with diabetes mellitus: an analysis of implementation in the SUS in Recife (PE). Saúde Debate. 2015 Dec;39(especial):268-82. http://dx.doi.org/10.5935/0103-1104.2015S005368.

29. Soranz D, Felipe Pinto L, Camacho LAB. Analysis of the attributes of primary health care using the electronic medical records in the city of Rio de Janeiro. Ciênc Saúde Coletiva. 2017 Mar;22(3):819-30. http://dx.doi.org/ 10.1590/1413-81232017223.33142016.
Copyright $\odot 2019$ Revista Latino-Americana de Enfermagem Este é um artigo de acesso aberto distribuído sob os termos da Licença Creative Commons CC BY.

Esta licença permite que outros distribuam, remixem, adaptem e criem a partir do seu trabalho, mesmo para fins comerciais, desde que Ihe atribuam o devido crédito pela criação original. É a licença mais flexível de todas as licenças disponíveis. É recomendada para maximizar a disseminação e uso dos materiais licenciados. 